Planetary Systems in the Universe - Observation, Formation and Evolution

Proceedings IAU Symposium No. 202, (C)2004 IAU

Alan Penny, Pawel Artymowicz, Anne-Marie Lagrange, \& Sara Russell, eds.

\title{
Turbulence, Vorticity Generation and Angular Momentum Transport via the Baroclinic Instability in Accretion Disks
}

\author{
Hubert Klahr \\ UCO/Lick Observatory, Santa Cruz, CA 96064, USA
}

Peter Bodenheimer

UCO/Lick Observatory, Santa Cruz, CA 96064, USA

\begin{abstract}
We propose the global baroclinic instability as a source for vigorous turbulence leading to angular momentum transport in Keplerian accretion disks. We know from analytical considerations and three-dimensional radiation hydro simulations that, in particular, protoplanetary disks have a negative radial entropy gradient, which makes them baroclinic. Two-dimensional numerical simulations show that this baroclinic flow is unstable and produces turbulence. These findings were tested for numerical effects by performing barotropic simulations which show that imposed turbulence rapidly decays. The turbulence in baroclinic disks draws energy from the background shear, transports angular momentum outward and creates a radially inward bound accretion of matter, thus forming a self consistent process. Gravitational energy is transformed into turbulent kinetic energy, which is then dissipated, as in the classical accretion paradigm. We measure accretion rates in $2 \mathrm{D}$ and $3 \mathrm{D}$ simulations of $\dot{M}=-10^{-9}$ to $-10^{-7} \mathrm{M}_{\odot} \mathrm{yr}^{-1}$ and viscosity parameters of $\alpha=10^{-4}-10^{-2}$, which fit perfectly together and agree reasonably with observations. The turbulence creates pressure waves, Rossby waves, and vortices in the $(R-\phi)$ plane of the disk. We demonstrate in a global simulation that these vortices tend to form out of little background noise and to be long-lasting features, which have already been suggested to lead to the formation of planets.
\end{abstract}

\section{Introduction}

Protoplanetary disks appear to be a common feature around young stars. They are thought to provide the material and the environment for the formation of planets. Thus one needs to know the internal properties of such disks, such as the density, temperature and turbulence, in order to estimate the time scales of the formation process. These quantities are not directly accessible via observation, and so one needs a model for these disks to derive observable quantities like line emission and scattering efficiency for the light from the central star.

The basic idea of most models is that there is a process in these disks that transfers angular momentum radially outward, so that mass will flow radially inward. Such a process might be turbulence (hydrodynamical or magnetohydrodynamical) or self gravity. Independent of the source for the angular momentum transport, it can be parameterized by an effective viscosity $\nu$, which usually is scaled to the local sound speed $c_{s}$ and the pressure scale height $H_{D}$ of the disk by a dimensionless number called $\alpha$ with $\nu=\alpha c_{s} H_{D}$. Despite the 


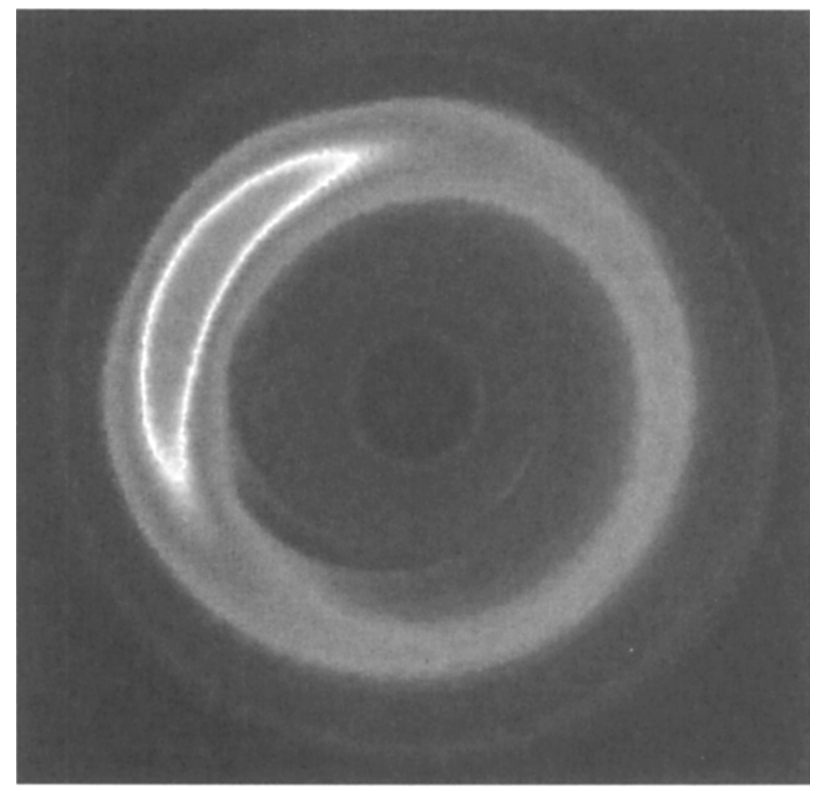

Figure 1. A "pre-protoplanet": Surface density (colors: 500 [red], 400 [yellow], 300 [green], to 100 [blue] $\mathrm{g} / \mathrm{cm}^{2}$ ) in a global model (1-10 AU in radius) projected in a cartesian frame after 567 orbits at the outer radius, which corresponds to $\approx 2 \times 10^{4} \mathrm{yr}$.

success of these models, $\alpha$ is still a free parameter for protoplanetary accretion disks. A barotropic Keplerian shear flow is in principle stable (Rayleigh stable) and does not develop turbulence per se despite the high Reynolds numbers. Hydrodynamical turbulence has shown to transport angular momentum outward (Hawley, Balbus, \& Winters 1999), but it is not able to sustain itself and usually rapidly decays in barotropic simulations. Only in the vicinity of the star, where dust has evaporated and no planets can form, is there sufficient ionization for the development of magnetic turbulence, which can generate $\alpha \approx 0.01$.

Indeed, if there is no self gravity working, basically no ionization present and no shear instability possible, the disk would develop no turbulence, transport no angular momentum, release no accretion energy and would basically cool down to the ambient temperature, while orbiting the star on a time-constant orbit. In such a disk, time scales for the growth of planetesimals would be much longer than in the standard scenarios, as the Brownian motion of the micron-sized dust is smaller and there is no turbulence acting on the dust in the $\mathrm{cm}$ - to $\mathrm{m}$-size range.

In Klahr \& Bodenheimer (2001) we present numerical simulations of a purely hydrodynamical instability that works in accretion disks, namely the well known baroclinic instability, which is also responsible for turbulent patterns on planets, for example, Jupiter's red spot and the weather patterns of cyclones and anti-cyclones on earth. Baroclinic instabilities arise in rotating fluids when surfaces of constant density are inclined with respect to the surfaces of constant pressure (e.g. Tritton \& Davies 1985). Vortensity, defined as vorticity per unit 
surface density, is not conserved as is the case in barotropic two-dimensional flows, and vortices can be generated.

We observe a global baroclinic instability that arises from the general radial stratification of the gas flow in accretion disks. Our motivation is based on the observation of positive Reynolds stresses in radiation-hydrodynamical threedimensional (3D) simulations of thermal convection in protoplanetary accretion disks (Klahr \& Bodenheimer 2000a). 2D-Models with radially varying entropy gradient show the generation of turbulence, while models without (e.g. globally isothermal models) are perfectly stable as expected. Also the 2-D tests show that indeed there is angular momentum transport outwards and that the lower-order azimuthal modes give the fastest growth rates in baroclinic simulations.

\section{Conclusions}

The global baroclinic instability is found to generate turbulence in disks and drive an accretion process.

In general we found numerically that isotropic turbulence in the $R-\phi$ plane of the disk has the property of transporting angular momentum radially outward. But only the global baroclinic instability seems to provide a reliable source for this turbulence in the first place. Thermal convection in the vertical direction of the disk is not necessary for this effect.

Thermal convection is only indirectly related to the baroclinic instability, as convective and radiative transport are responsible for the radial temperature distribution and therefore the radial entropy gradient.

The anti-cyclonic rotating gas parcels (see Fig. 1) are vortices, that could be the precursors of planetary formation. They can be thought of as preprotoplanets.

\section{References}

Hawley, J., Balbus, S.A. \& Winters, W.F. 1999, ApJ, 518, 394

Klahr, H.H. \& Bodenheimer, P. 2000a, Proceedings of: Disks, Planetesimals and Planets, ASP Conference Series, eds. F. Garzn, C. Eiroa, D. de Winter \& T. J. Mahoney (Astronomical Society of the Pacific)

Klahr, H.H. \& Bodenheimer, P. 2001, ApJ, submitted

Tritton, D.J. \& Davies, P.A.1985, in Hydrodynamical Instabilities and the Transition to Turbulence, ed. H.L. Swiney \& J.P. Gollub (Berlin: Springer-Verlag), 229 